\title{
Créer les conditions du succès de la filière industrielle française des thérapies innovantes
}

\author{
Pierre Noel Lirsac ${ }^{1}$, Olivier Blinn², Jérémy Magalon ${ }^{3,4}$, et les participants à la table ronde $N^{\circ} 5$ de Giens XXX: \\ Pierre Angot ${ }^{5}$, Estelle de Barbeyrac ${ }^{6}$, Pascal Bilbault ${ }^{7}$, Elisabeth Bourg ${ }^{8}$, Odile Damour ${ }^{9}$, Patrick Faure ${ }^{10}$, \\ Nicolas Ferry ${ }^{11}$, Bénédicte Garbil ${ }^{5}$, Jérôme Larghero ${ }^{12}$, Marina Nguon $^{12}$, François Pattou ${ }^{13}$, Stéphane Thumelin ${ }^{14}$ \\ et Frank Yates ${ }^{15 \dagger}$ \\ 1 CELLforCURE, Groupe LFB, Les Ulis, France \\ 2 Assistance Publique-Hôpitaux de Marseille, Pharmacologie Clinique, CHU Timone, Marseille, France \\ 3 Laboratoire de Culture et Thérapie Cellulaire, Assistance Publique des Hôpitaux de Marseille, Marseille, France \\ 4 Vascular Research Center Marseille, Inserm UMRS 1076, Faculté de Pharmacie, Marseille, France \\ 5 Ministère de l'Economie, de l'Industrie et du Numérique, Paris, France \\ 6 Genethon, Evry, France \\ 7 Laboratoire Boehringer Ingelheim, Reims, France \\ 8 Laboratoire Novartis, Rueil Malmaison, France \\ 9 Hospices Civils de Lyon, Lyon, France \\ 10 SATT Sud Est, Marseille, France \\ 11 ANSM, Saint Denis la Plaine, France \\ 12 Assistance Publique-Hôpitaux de Paris, Paris, France \\ 13 Centre Hospitalier Régional Universitaire, Lille, France \\ 14 Inserm Transfert, Paris, France \\ 15 Sup'Biotech/CEA, Montrouge, France
}

Texte reçu le 18 novembre 2014 ; accepté le 24 novembre 2014

\begin{abstract}
Mots clés :
médicaments

Résumé - Alors que l'Union Européenne n'avait fait que suivre les initiatives des Etats-Unis et du Japon en introduisant de thérapies innovantes ; des régimes particuliers pour les médicaments orphelins, elle a instauré un statut particulier à une nouvelle catégorie de médicaments de thérapies innovantes pour un patient ; organisme génétiquement modifié ; accès au marché médicaments biologiques, les médicaments de thérapie innovante (MTI) et y associe des règles spécifiques. Le règlement européen (qui constitue l'instrument juridique le plus élevé dans la hiérarchie des textes du droit européen) CE n 1394/2007, publié en 2007 définit sous ce terme les médicaments de thérapie cellulaire somatique, les médicaments issus de l'ingénierie tissulaire, et les médicaments de thérapie génique, éventuellement couplés à des dispositifs médicaux. L'objectif affiché était double : à la fois promouvoir leur industrialisation et leur accès au marché tout en garantissant un niveau élevé de protection sanitaire aux patients. Depuis la publication du règlement, les autorisations de mise sur le marché délivrées en Europe sont peu nombreuses et ne sont pas accompagnées par un succès commercial. Cependant, certaines études récentes montrent qu'il s'agit d'un secteur en croissance et que la France reste la première nation européenne en termes de nombre d'essais cliniques. Cette table ronde a réuni un panel de représentants d'acteurs publics et privés français du secteur des thérapies innovantes. Les discussions ont été focalisées sur les conditions du succès de la recherche translationnelle et de manière plus globale, de la filière française des thérapies innovantes. Elles ont conduit à identifier un certain nombre de verrous dont la levée, formulée sous forme de recommandations, permettrait de faciliter le développement et le succès de cette filière.
\end{abstract}

Abréviations : voir en fin d'article.

\footnotetext{
$\dagger$ Les articles, analyses et propositions des Ateliers de Giens n’engagent que leurs auteurs et ne préjugent pas de leur organisme de tutelle.
} 


\section{Introduction}

\section{1. Etat des lieux réglementaires}

Le règlement européen CE n ${ }^{\circ}$ 1394/2007, publié en 2007, a défini une nouvelle catégorie de médicaments biologiques : les médicaments de thérapie innovante (MTI). Ils regroupent les médicaments de thérapie cellulaire somatique, les médicaments issus de l'ingénierie tissulaire, et les médicaments de thérapie génique, éventuellement couplés à des dispositifs médicaux. Jusqu'en 2007, seule la France disposait d'une réglementation. Jusqu'à présent, ces produits thérapeutiques étaient des préparations de thérapies cellulaires, tissulaires (PTC) ou de thérapies géniques à finalité thérapeutique et n'étaient pas considérés comme des médicaments au sens du code de la Santé Publique. Cependant, ils restaient sous la compétence de l'Agence Nationale de Sécurité du Médicament et des Produits de Santé (ANSM) et réglementés au niveau national sur la base de la directive tissus/cellules (directive 2004/23/CE). Ils étaient produits par des établissements autorisés qui pouvaient être des hôpitaux, l'Etablissement Français du Sang ou des industries pharmaceutiques et ne répondaient pas aux bonnes pratiques de fabrication (BPF) «médicaments ». Désormais, un produit dont le procédé de fabrication ne fera pas appel à des modifications substantielles ET dont la destination sera la même chez le donneur et le receveur restera une préparation cellulaire ou tissulaire. Si l'une de ces deux conditions n'est pas vérifiée, il sera considéré comme un MTI. Il en résulte qu'en dehors des greffes conventionnelles de cellules souches hématopoiétiques et de la greffe d'îlots de Langerhans, la majorité des produits de thérapies innovantes développés en France, répond désormais à la définition d'un MTI et a donc changé de cadre réglementaire.

Les MTI étant des médicaments, leur commercialisation nécessite au préalable l'obtention d'une autorisation de mise sur le marché (AMM). Cette dernière relève de la procédure centralisée et est délivrée par la Commission Européenne après évaluation de l'Agence Européenne du Médicament (EMA). Les conditions de production et de distribution des MTI doivent être conformes aux BPF. En Europe, tout établissement conforme aux BPF peut préparer des médicaments dès lors qu'il bénéficie aussi d'un statut d'établissement pharmaceutique mais en France les établissements de santé ne peuvent bénéficier du statut d'établissement pharmaceutique. Les MTI sont soumis au principe de libre circulation des marchandises et peuvent donc circuler au sein de l'espace économique européen hors du pays hébergeant la structure de production et leur surveillance post-AMM relève de la pharmacovigilance.

Pour les MTI préparés ponctuellement (MTI-PP) pour un patient donné, et utilisés au sein du même Etat-membre, l'article 28 du règlement européen définit l' « exemption hospitalière » qui relève d'une autorisation nationale. En d'autres termes, les MTIPP ne sont pas assujettis aux exigences du règlement 1394/2007 et ne peuvent faire l'objet d'une demande d'AMM centralisée ; ils suivent un cadre réglementaire national qui doit être équivalent aux règles communautaires applicables en matière de qualité et de sécurité.

En France, ce règlement européen qui est d'application immédiate conformément au droit européen, a entraîné des modifications du code de Santé Publique français suite à la publication de la loi $\mathrm{n}^{\circ}$ 2011-302 du 22 mars 2011. Cette loi a ensuite été déclinée par le décret $n^{\circ}$ 2012-1236 du 6 novembre 2012 relatif aux MTI, et introduisant la notion d'exemption hospitalière définie par le terme médicaments de thérapie innovante préparés ponctuellement (MTI-PP). Si les MTI doivent être fabriqués dans un établissement pharmaceutique privé, public ou créés au sein d'organismes à but non lucratif mais autres que les établissements de santé, les MTI-PP peuvent également être fabriqués par des structures au sein d'établissements de santé.

C'est dans ce contexte que de nouvelles structures dédiées à la production de MTI en France ont récemment vu le jour :

- d'une part, deux structures de production industrielles uniques en Europe :

- CELLforCURE, filiale du LFB et dont la plateforme de production industrielle de MTI à très grande échelle pour les phases cliniques tardives et le marché, a été inaugurée aux Ulis le 10 septembre 2013. La mise en place de cette plateforme s'inscrit dans le cadre du projet $\mathrm{C} 4 \mathrm{C}$ et a reçu le soutien du programme des investissements d'avenir et de BpiFrance (ex-Oséo) en 2012. Le montant total de l'investissement s'élève à 80 millions d'euros incluant le soutien par BpiFrance au développement de 5 projets portés par des petites et moyennes entreprises (PME) et acteurs académiques jusqu'au marché pour un montant de $20 \mathrm{M} €$,

- Genethon, le laboratoire de l'Association Française contre les Myopathies (AFM)-Téléthon a obtenu de l'ANSM le statut d'établissement pharmaceutique le 27 juin 2013. Son centre de production, Genethon Bioprod est ainsi autorisé à produire des MTI. Le coût de la construction de Genethon Bioprod s'élève à 28,5 millions d'euros dont 5,5 financés par l'AFM, 8 par le Conseil Régional d'Ile de France, 7 par le Conseil Général de l'Essonne et 8 par Génopole d'Evry. Son coût de fonctionnement annuel (environ 10 millions d'euros) est intégralement financé par l'AFM grâce aux dons du Téléthon.

- À ces structures industrielles, viennent s'ajouter :

- des acteurs publics comme l'Etablissement Français du Sang (EFS) et l'Établissement de Santé des Armées. Pour l'EFS, Atlantic bioGMP (ABG), situé près de Nantes, a obtenu le 6 février 2014 le statut d'établissement 
pharmaceutique, pour son activité de production de MTI. En matière de financement, l'EFS, Nantes métropole, le Conseil Régional de Pays de la Loire, le Conseil Général de Loire Atlantique et le Fonds Européen de Développement Régional (FEDER [Union Européenne]) ont participé à titre d'investissement à hauteur de 5,2 millions d'euros. Les frais de fonctionnement d'ABG sont assurés à hauteur de 1,5 millions d'euros par an par l'EFS, l'AFM, l'Institut National de la Santé et de la Recherche Médicale (Inserm) et le Centre Hospitalier Universitiaire (CHU) de Nantes.

\subsection{Les thérapies innovantes: un marché émergent en forte croissance}

La croissance économique du secteur des thérapies innovantes est la résultante de deux évènements interdépendants :

- d'une part, des découvertes scientifiques majeures dans ce domaine dans les deux dernières décennies, allant de la découverte des cellules souches embryonnaires en 1998 par James Thomson, ${ }^{[1]}$ au succès des greffes de thérapie génique pour guérir les bébés bulles dans les années $2000^{[2]}$ jusqu' à la technologie de reprogrammation cellulaire (cellules pluripotentes induites [iPS]) mise au point par Yamanaka en 2007 (prix de Nobel de médecine en 2012 avec John Gurdon). ${ }^{[3]}$ Plus récemment, l'utilisation avec succès du chimeric antigen receptor (CAR) dans des leucémies pédiatriques ${ }^{[4]}$ ou l'inclusion du premier patient traité à partir d'iPS dans un essai clinique au Japon laissent entrevoir des perspectives florissantes pour ce secteur;

- d'autre part, l'application du règlement européen dont les objectifs sont :

- de faciliter et stimuler la recherche sur ces nouvelles approches du fait d'un cadre harmonisé au niveau européen,

- d'assurer un accès au marché de l'ensemble des Etats-membres de l'Union Européenne via une AMM centralisée,

- de stimuler la recherche et le développement de ces produits par la mise en place de mesures incitatives.

Deux études récentes ont été menées à l'initiative de CELLforCURE afin de répertorier l'ensemble des essais cliniques utilisant des MTI dans le monde (à l'exception du Japon), tels qu'ils apparaissent dans les bases de données des essais cliniques des agences nationales et à travers l'analyse des communications publiques, menées respectivement en 2013 et 2014 :

- la première constatation porte sur le fait que leur nombre a doublé entre juin 2013 (2 740) et juin 2014 (4 760);
- la seconde constatation concerne le nombre d'approches allogéniques décrites dans ces essais cliniques, qui a doublé entre 2013 et 2014. En effet, d'un point de vue pharmaceutique, le MTI allogénique se rapproche d'une bioproduction industriellement plus classique, fondé sur une « augmentation d'échelle » (scale-up) permettant de traiter de larges populations de patients, mais présentant des questionnements médicaux (compatibilité) qui restent à surmonter. Inversement, les MTI autologues et certains MTI allogéniques dont chaque lot produit est administré à un unique patient receveur, se rapprochent en terme de ratio bénéfice-risque des greffons de cellules, tissus ou organes

- enfin, la répartition géographique des MTI en nombre de développements cliniques montre toujours qu'une part prépondérante de l'activité dans ce domaine revient aux Etats-Unis (58 \%). Cependant, la France est leader en Europe, devant l'Allemagne, l'Espagne et l'Italie (figure 1).

L'industrialisation du secteur des MTI semble logiquement influencer l'économie du secteur et les différentes évaluations du marché potentiel des MTI au niveau mondial sont respectivement à l'horizon 2016 de 8,8 milliards de dollars et de 20 milliards de dollars à l'horizon 2020 dont 2 pour l'Europe avec des taux annuels de croissance évalués entre 20 et $30 \%$. ${ }^{[5]}$

\subsection{Booster le développement de la filière industrielle française des thérapies innovantes}

Aujourd'hui, une grande part des produits pharmaceutiques en développement sont des biomédicaments sur un marché pharmaceutique mondial de plus de 1150 milliards de dollars. La France, historiquement à la pointe dans le domaine des médicaments issus de la chimie, prend le virage des biotechnologies en se positionnant sur un sujet d'avenir : les médecines personnalisées cellulaires et géniques.

Notre pays peut compter sur un modèle de recherche hospitalo-universitaire performant permettant d'associer au sein de plateformes (Centres d'Investigations Cliniques [CIC-biothérapies]) des compétences clinique, biologique, anatomopathologique provenant à la fois de chercheurs, ingénieurs, médecins, pharmaciens, avec un accès direct aux patients et aux matériels biologiques. A cette recherche académique performante, s'ajoutent des capacités de bioproduction industrielle importantes ainsi qu'un écosystème de PME de haute technologie émergent mais compétitif au plan international. La France a tous les atouts pour prendre une place de leader sur un des grands gisements de croissance des années et décennies à venir.

Conscient de ces enjeux, et pour ancrer savoir-faire, emplois et capacités au sein des acteurs du territoire, le gouvernement a 


\section{Pourcentage d'études par pays}

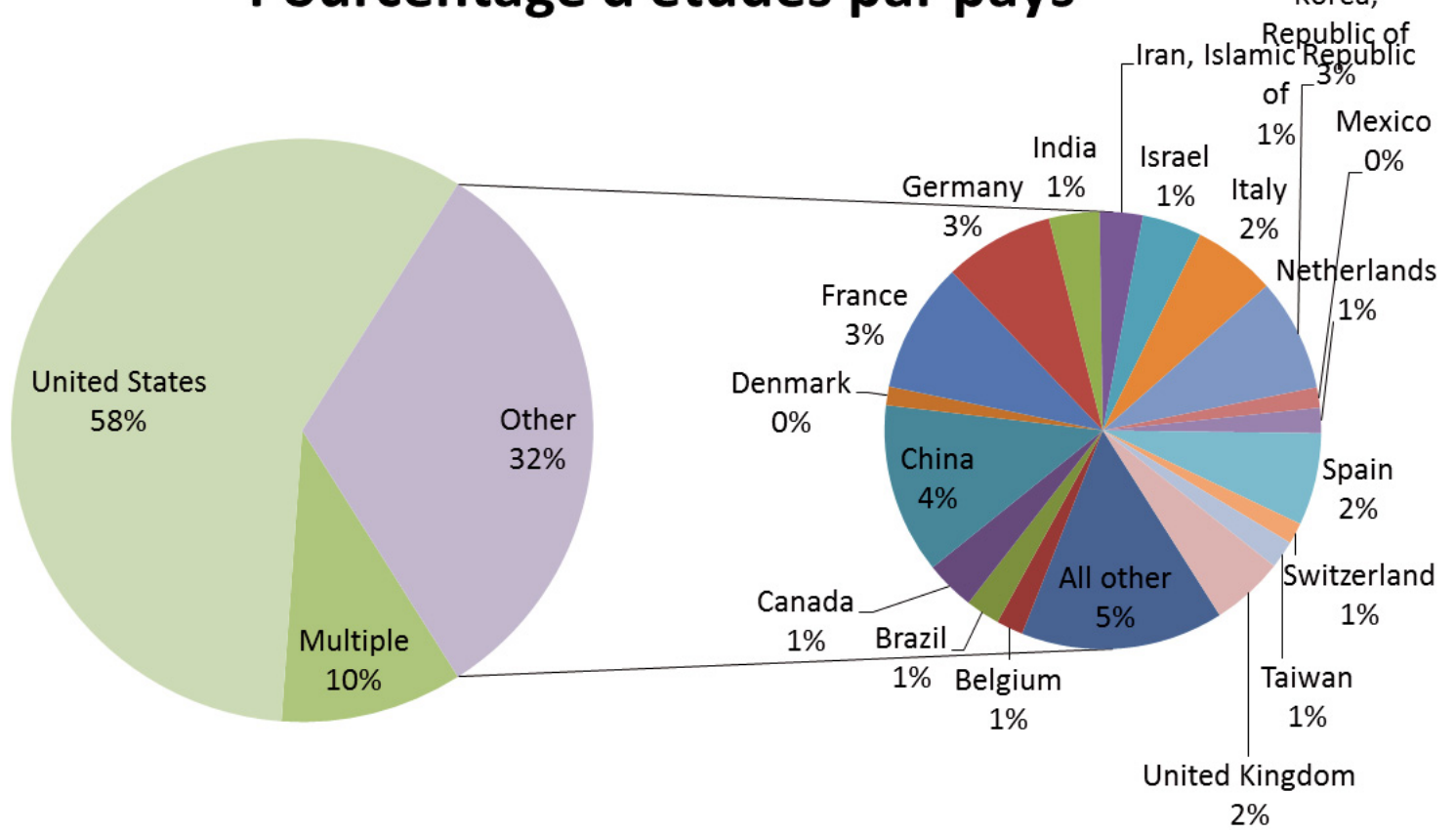

Fig. 1. Pourcentage d'études de médicaments de thérapies innovantes par pays en 2014. Données étude CELLforCure-IEEP (données non publiées, avec l'aimable autorisation de CellforCure).

décidé de soutenir la mise en place d'une filière industrielle française des thérapies innovantes, notamment à travers le dispositif des investissements d'avenir et la mise en place de deux platesformes industrielles uniques en Europe permettant la production en grade BPF pour les essais cliniques et le marché. Il faut associer à ces établissements la capacité à structurer, formaliser, conduire et financer les développements nécessaires dans le respect des exigences règlementaires, et les outils de BpiFrance de soutien aux développements des projets.

En parallèle, la France dispose d'acteurs nationaux et régionaux du transfert de technologies permettant d'orienter et d'accompagner une découverte scientifique vers le développement d'un produit de santé innovant, en particulier :

- Inserm-Transfert, filiale privée de valorisation de l'Inserm, possède un portefeuille de près de 1000 familles de brevets, licenciés pour moitié, permet de protèger et promouvoir depuis 2006, les savoir-faire, expertises scientifiques et cliniques et inventions issues des 290 unités de recherche de l'Inserm. En dépit d'un appétit industriel encore très modeste dans le secteur, Inserm-Transfert a anticipé depuis plusieurs années déjà la nécessité de renforcer la position française à travers la protection de futurs monopoles commerciaux dans les domaines de la thérapie génique, de la thérapie cellulaire et de la médecine régénérative plus particulièrement. Cette anticipation a été faite en ciblant des problèmes majeurs de santé publique (impasse thérapeutique, coût de prise en charge des patients...) auxquels ces approches thérapeutiques innovantes sont susceptibles d'apporter une solution à une échelle industrielle. La mise en place à un niveau national de projets de développement préindustriels d'envergure à vocation industrielle apparait être un relai nécessaire pour l'accélération du développement de la filière industrielle sur le territoire national à côté des initiatives locales hospitalo-universitaires ;

- au côté de structures nationales de valorisation comme l'Inserm-transfert, les Sociétés d'Accélération du Transfert de Technologies (SATT) ont été créées en région et sont financées dans le cadre du programme d'investissements d'avenir. Les SATT accompagnent le transfert de technologies innovantes issues de la recherche publique vers le monde économique par la concession de licences d'exploitation sur des titres de propriété intellectuelle à des entreprises. Aujourd'hui, 160 établissements de recherche publics ont confié la valorisation de leurs résultats de recherche aux 14 SATT couvrant le territoire. A leur actif : 2900 projets détectés, 540 brevets déposés, $70 \mathrm{M} €$ investis dans des maturations juridique (propriété intellectuelle), économique (marché) et technologique (preuve de concept), 140 licences signées et 40 start-up créées.

Aujourd'hui, un certain nombre de verrous pèsent encore sur le développement de cette filière industrielle et sur la translation des projets innovants vers l'aval que constituent les développements cliniques, la production industrielle et l'accès au marché. Ces difficultés sont analysées ci-après. 


\section{Méthode}

Pour cette table ronde, le groupe associait tous les acteurs de la filière : des membres du milieu académique (5 personnes), des industriels ( 3 personnes), des acteurs du transfert de technologie ( 2 personnes), un représentant des agences réglementaires (ANSM), un représentant du Ministère de l'Economie, de l'Industrie et du Numérique et un représentant du monde enseignant.

Une réunion préparatoire a permis de définir le déroulement et l'organisation de la table ronde en différentes sessions successivement consacrées à :

- la présentation de cas pratiques et de retours d'expérience de la part des représentants académiques concernés par le nouveau règlement européen ;

- une session dédiée aux attentes et au retour d'expérience des acteurs industriels ;

- l'intervention des acteurs de transfert de technologie des organismes de financement et aux attentes et à la vision des autorités sanitaires et du Ministère de l'Economie de l'Industrie et des Technologies Numériques;

- la discussion et la rédaction des constats et recommandations.

Ces nombreuses présentations et les discussions qui en ont découlé ont démontré l'intérêt du développement rapide et efficient d'une filière industrielle française des thérapies innovantes. Néanmoins, nous avons identifié un certain nombre de questions et de verrous au développement de cette filière émergente. Les réflexions et propositions de la table ronde sur ces différents points sont détaillées ci-après.

\section{Verrous et recommandations}

\subsection{Communication}

\section{- Une perception floue et pleine d'a priori du secteur des thérapies innovantes}

La perception a priori des thérapies innovantes est celle d'un marché de niche, soulevant des questions relatives à la sécurité. La complexité médico-technique est perçue comme génératrice de coûts et comme frein au développement. De plus, les efforts de vulgarisation et de communication vers le grand public sont insuffisants et délivrés au coup par coup, ce qui renvoie le public à la perception d'une technologie qui relèverait de l'exploit technologique ou du traitement exceptionnel, contribuant à une distanciation par rapport à ces nouvelles thérapies.

Il est évident que les avancées technologiques vont permettre de diminuer les coûts. Les exigences règlementaires étant les mêmes, la sécurité est identique à celle des autres médicaments. Seul le recul manque encore.
Des efforts financiers conséquents ont été consentis en France pour mettre en œuvre les protocoles de thérapie cellulaire dans les établissements hospitaliers. Cependant, ces initiatives souffrent d'un manque de visibilité. Les registres institutionnels des essais cliniques français ${ }^{[6]}$ pointent vers des données partielles et peu à jour, notamment en comparaison avec les données obtenues sur le site américain «ClinicalTrials » maintenu par le National Institute of Health (NIH). ${ }^{[7]}$ Par exemple, une recherche "gene therapy" restreinte à la France sur ClinicalTrials.gov renvoie 276 réponses tandis qu'une requête similaire sur le site "Essais cliniques de médicaments » en renvoie 61 (dont le dernier date de 2011).

Ce manque de visibilité et la difficulté à obtenir des informations sur les essais en cours peut ainsi être interprété par certains comme un manque de transparence. D'autre part un industriel qui souhaiterait connaitre les leaders français dans le domaine des essais cliniques en thérapies innovantes n'aurait donc à travers les outils disponibles en France qu'une image partielle du dynamisme du pays en la matière et ne disposerait pas des contacts institutionnels les plus pertinents pour développer un éventuel partenariat.

En termes de communication et de vulgarisation, un réel effort de communication vers le grand public est consenti par les partenaires institutionnels, qui se traduit par des communiqués de presse qui peuvent être repris par la presse généraliste. ${ }^{\left[{ }^{8,9]}\right.}$ Néanmoins, ces communiqués, émanant d'agences ayant des politiques de communication différentes, mériteraient d'être rassemblés et mis en valeur de manière centralisée.

\section{Propositions}

Améliorer la perception et la compréhension des thérapies innovantes par le grand public : demander au porteur du plan biotechnologies médicales «l'humain réparé » ${ }^{[10]}$ de communiquer sur l'intérêt sociétal de ces nouvelles technologies, sur la base de quelques success stories, et de pousser les acteurs à communiquer sur les patients reports pertinents pour le grand public.

Améliorer la visibilité des essais cliniques en cours : dans un souci de visibilité et de compétitivité, et compte tenu du dynamisme de la France dans ce domaine, il est important que l'ANSM mette à jour le lien renvoyant vers le répertoire des essais cliniques afin d'obtenir un niveau d'information similaire à celui de pays comparables.

\subsection{Technologiques et médicaux}

\section{- Capacité technologique à industrialiser à des coûts acceptables, les procédés de production de MTI}

Pour les procédés basés sur des technologies allogéniques avec scale up (1 donneur pour $\mathrm{n}$ receveurs), le procédé de production se fonde sur des techniques similaires à celles utilisées en 
lignées pour produire des protéines recombinantes à l'échelle du laboratoire, de quelques litres à plusieurs dizaines de litres. Dans le cas de produits autologues, il s'agit de produire un médicament pour un patient à partir de ses propres cellules, généralement préparé en urgence à proximité. Cette dernière approche modifie totalement nos modes actuels de production et nous amène aujourd'hui à imaginer l'industrialisation de la thérapie cellulaire.

Cette perspective impose d'importants investissements matériels. Dans le cas des cultures cellulaires, des développements de systèmes jetables sont en cours et ces nouveaux modes de fabrication vont imposer des contrôles individuels des cultures. Cela pose en particulier la question de la miniaturisation de la mesure et du contrôle des divers paramètres physico-chimiques tout au long des cultures. Les technologies de mesure sont aujourd'hui conçues pour des gros bioréacteurs mais requièrent encore d'être miniaturisées, jetables et de faible coût. Il y a vraisemblablement là une rupture de conception. Il faut revoir l'ensemble des systèmes de mesure des paramètres physico-chimiques des cultures avec des senseurs miniaturisés et jetables après chaque manipulation dans un cadre de BPF. Aujourd'hui, ceci n'est pas encore réalisable à un coût raisonnable.

Nous sommes face à un vrai challenge autour de l'électronique et de la micro-électronique. Les équipements d'aujourd'hui reposent le plus souvent sur des solutions étrangères le plus fréquemment allemandes ou américaines. Ceci conduit les industriels à établir des partenariats, pour gagner du temps et s'appuyer sur les meilleures solutions disponibles. Cependant il est possible de développer d'autres solutions qui pourraient être françaises, car nous avons en France (notamment à Grenoble) des experts de la microélectronique. Il y a là des champs d'opportunité en capitalisant sur des savoir-faire de laboratoires publics (Commissariat à l'Energie Atomique et aux Énergies Alternatives [CEA], Centre National de la Recherche Scientifique [CNRS], ..) et des industriels.

\section{Propositions}

Traduire dans la programmation de l'Agence Régionale de la Recherche (ANR) la politique volontariste de développement de solutions technologiques françaises : les entreprises françaises qui développent les technologies nouvelles de la médecine personnalisée cellulaire et génique vont produire les médicaments de demain. Pour que la production s'accélère, il faut que la technologie permette de les réaliser dans le des BPF. Il faut aussi une politique volontariste du Ministère de l'Enseignement Supérieur et de la Recherche pour développer des solutions françaises dans ce domaine. En particulier la programmation de l'ANR devrait promouvoir spécifiquement le développement de solutions métrologiques miniaturisées et jetables adaptées à l'échelle autologue capitalisant sur les compétences nationales existantes en micro- et nano-technologies.

\section{- Sécuriser l'utilisation des produits allogéniques et des thérapies basées sur les cellules souches pluripotentes induites (iPS) en clinique}

L'utilisation de MTI allogéniques est un défi en soi, qui permettra d'utiliser les cellules d'un patient pour en traiter plusieurs centaines, voire des milliers. Ce modèle est fondamentalement un modèle de production industrielle de médicament proche de ceux utilisés pour produire les médicaments recombinants. Ceci peut naturellement permettre de diminuer considérablement les coûts de revient unitaires des traitements.

Cette perspective est encore au début des stades cliniques avec différentes approches. Mais avant d'être accessible au patient il importe de résoudre deux questions :

- la première est d'ordre médical impliquant l'acceptabilité par l'organisme des patients de cellules d'un autre individu. C'est toute la difficulté de l'immunosuppression qui peut être atténuée par l'utilisation de types cellulaires peu immunogènes ou ayant des propriétés immuno-modulatrices;

- la deuxième est liée aux systèmes cellulaires utilisés pour obtenir des cellules progénitrices. Aujourd'hui, on extrait les cellules souches d'un prélèvement biologique (p. ex. sang de cordon ou prélévement de moelle, biopsie ...) mais ce n'est pas la seule solution. Avec les cellules souches pluripotentes induites (ou iPS) [prix Nobel 2012] on peut désormais prendre n'importe quelle cellule d'un organe (peau ...), la manipuler pour la dédifférencier et lui donner la pluripotence nécessaire avant de la re-spécialiser en cellules ou tissus médicaments. C'est effectivement l'une des solutions pour la médecine régénérative de demain, que ce soit pour des stratégies allogéniques ou autologues, dès lors que l'on aura levé les incertitudes sur la sécurité biologique de ces systèmes à long terme et leur potentialité à long terme. Le premier essai clinique a débuté au Japon dans la dégénérescence maculaire liée à l'âge (DMLA).

Des acteurs académiques français sont à la pointe dans ce domaine, et au niveau industriel, la France dispose d'entreprises capables de répondre à ce défi. La priorité est aujourd'hui au développement d'une approche nationale structurée de recherche en amont :

i. sur les ressorts de la pluripotence cellulaire et de la différenciation cellulaire ;

ii. sur le comportement in vitro versus in vivo des cellules souches et des cellules différenciées dérivées afin de mieux comprendre les mécanismes de différenciation et le devenir des cellules, et pouvoir mieux faire le lien entre les modèles et la clinique. 
Pour mémoire, l'ANR a mis en place dès 2010 un appel à projet « programme recherches partenariales et innovation biomédicale » (RPIB) qui inclut les cellules souches et a réalisé dès 2010 un appel à projets de recherche finalisée sur les cellules souches. Même si elles existent, ces initiatives restent d'ampleur anecdotique, sont ponctuelles et ne sont pas structurées dans la durée. La structuration de cette approche devrait être impulsée par les ministères concernés, à travers un plan pluriannuel de 3 à 5 ans, soutenu par des appels d'offres idoines.

\section{Propositions}

Traduire dans la programmation de l'ANR l'importance des études relatives à la sécurité des thérapies innovantes : l'État doit mettre en place une ligne spécifique dans la programmation de l'ANR soutenant les études relatives à la sécurité des thérapeutiques innovantes (iPS). Elle permettra ainsi aux acteurs académiques français qui sont à la pointe dans ce domaine, de développer des projets ciblés sur les ressorts de la pluripotence cellulaire et la différenciation cellulaire et sur le comportement in vitro versus in vivo des cellules souches et des cellules différenciées dérivées.

\section{- Les équipes hospitalo-universitaires sont en première position en matière de développement de MTI-PP, mais l'accès aux patients et au transfert de technologie reste un parcours long et difficile, faute d'accompagnement adapté}

Les acteurs hospitaliers sont en première ligne dans le domaine des thérapies innovantes où ils participent activement à trouver des solutions aux pathologies en impasse thérapeutique.

Différents types de PTC, MTI-PP et MTI sont développés par les équipes hospitalo-universitaires. Certaines structures hospitalo-universitaires sont bien adaptées à la production de PTC, mais le développement de MTI ou de MTI-PP fait appel à de plus larges compétences, dont certaines sont insuffisamment ou non présentes dans ces équipes, ni au sein de leur organisme de tutelle. Ces aspects sont clairement susceptibles de limiter leur participation de manière efficiente à la filière des MTI.

Pallier le manque de compétence en propriété intellectuelle (PI) et transfert industriel (TI) : le chercheur hospitalier travaille sur des solutions au problème de ses patients sans penser au TI et sans forcément penser à la PI. Il ne sait pas qu'il est aussi un maillon important de la valorisation industrielle qui va jusqu'à la création d'emploi. De plus, pour de nombreuses raisons et sous diverses pressions, il communique et publie le plus rapidement possible ses résultats sans penser à la PI qui constitue à ses yeux une source de difficultés administratives entraînant une perte de temps et ralentissant la publication.

\section{Propositions}

Développer une culture de la PI comme outil de valorisation : les organismes de transfert doivent sensibiliser les acteurs académiques sur la propriété intellectuelle en leur faisant comprendre que l'article scientifique rédigé sert au dépôt de brevet et que sa publication vient juste après, sans retard mais au contraire avec une véritable valorisation pour l'auteur comme pour son institution. Il doit se savoir libérer de tous les problèmes administratifs par les organismes tels que la SATT.

Accroitre la compétence nécessaire aux développements pharmaceutiques (procédé de fabrication et stratégie de contrôle qualité) et non-cliniques : les chercheurs hospitaliers ont un niveau d'expertise très élevé concernant la connaissance scientifique des produits qu'ils développent et peuvent ainsi concevoir de nouvelles solutions thérapeutiques. Ils ont également un haut niveau de connaissance scientifique concernant les modèles animaux ainsi que les compétences chirurgicales pour réaliser des études non-cliniques d'efficacité au sein de leur académie. En revanche, i) formaliser un procédé de fabrication pérenne associé à une stratégie de contrôle et ii) concevoir un plan de développement non-clinique, qui soient à la fois pertinents pour ces médicaments et conformes aux exigences pharmaceutiques, fait appel à d'autres champs de connaissance et de compétences. En effet, un certain nombre de guides (guidelines) ont été conçus par l'EMA concernant le développement non-clinique des médicaments de thérapie cellulaire, tissulaire ou génique, en plus des guidelines de l'International Conference on Harmonisation of Technical Requirements for Registration of Pharmaceuticals for Human Use (ICH) plus anciennes. L'interprétation et l'incorporation de ces recommandations nécessitent le dialogue avec d'autres types d'experts. De plus, une des conséquences de l'application du cadre réglementaire des médicaments est qu'il est demandé de réaliser les études toxicologiques dans des structures travaillant en conformité avec les bonnes pratiques de laboratoire (BPL). Cela pose le problème du financement de ces études qui doivent être externalisées dans des structures spécialisées et qui peuvent représenter un coût très important. Par faute de moyen et méconnaissance, les essais précliniques de toxicologie ne sont actuellement pas forcément réalisés avec des organismes qui répondent aux BPL et doivent bien souvent être refaits lors du transfert industriel.

\section{Propositions}

Accompagner la recherche académique en thérapies innovantes : mettre en place sous l'égide des SATT une structure mutualisée apportant les compétences nécessaires pour accompagner les académiques dans le développement de leur projet. Cette structure apportera toutes les compétences, dans le domaine de la qualité (documentation, audits, certificat ...), la préparation des dossiers réglementaires, le développement pharmaceutique du produit, marketing et trouvera l'industriel intéressé par le développement. De la même manière, il est nécessaire de favoriser les réunions d'échange entre acteurs académiques et autorités de 
santé afin de développer une meilleure connaissance des processus et exigences règlementaires.

\subsection{Réglementaires et législatifs}

\section{- Certaines spécificités réglementaires françaises obèrent le potentiel d'innovation académique de la filière}

L'une des particularités de la réglementation française applicable aux médicaments, est le monopole de leur fabrication par des établissement pharmaceutique (EP) et la non-éligibilité des établissements publics de santé au statut d'EP. Les autorités réglementaires françaises ont cependant pris en compte le fait que les fabricants sont le plus souvent des petites structures voire des structures du monde académique, et ont ainsi permis à de nouvelles structures de bénéficier du statut d'EP. Cela concerne notamment les organismes à but non lucratif ou les organismes publics (autres que les établissements de santé) qui étaient jusqu'alors exclus du champ des établissements pharmaceutiques. Pour l'instant, seuls les établissements de santé ne peuvent accéder au statut d'établissement autorisé à fabriquer des MTI, ce qui n'est pas leur mission première.

Par ailleurs, la loi tant au niveau européen (pour tous les médicaments, incluant les MTI) qu'au niveau national (pour les MTI-PP), impose que les médicaments soient fabriqués selon les principes des BPF. Concernant les MTI-PP, un projet de loi propose que les requis des BPF soient «adaptés » en fonction des types de médicaments fabriqués, sans donner plus de précisions. Il n'est notamment pour l'instant pas prévu par la loi que des établissements autorisés à fabriquer des MTI-PP puissent se voir décerner un certificat de conformité avec les BPF. Ceci est pour l'instant « réservé » aux établissement pharmaceutiques. Les hôpitaux français se trouvent en situation d'inégalité par rapport à d'autres pays européens qui eux, ont accès au certificat BPF pour produire des MTI et des MTI-PP sans avoir à recourir à des procédures complexes et avec une application plus souple et pragmatique de la réglementation pour ne pas freiner la dynamique car le respect des BPF pharmaceutiques en Europe est hétérogène. Cela est également susceptible de représenter un frein pour les MTI-PP qui seraient destinés à être transférés à des industriels pour poursuivre un développement à l'échelle européenne. L'Europe ne s'est pas encore prononcée sur la possibilité d'utiliser des données d'essais cliniques évaluant des MTI-PP pour une demande d'AMM, mais i) il sera en effet probablement plus difficile de faire reconnaitre à $100 \%$ la qualité et la conformité de ces médicaments auprès de l'Agence Européenne sans certificat BPF et ii) il apparait que dans le cas de maladies rares pour lesquelles le nombre de patients est faible, il n'y a pas de raison objective de ne pas prendre en considération ces données. Sous réserve bien sûr de justifier de cette stratégie et par exemple dans le cas d'une demande d'AMM conditionnelle qui permet de valider l'efficacité et la sécurité des médicaments par des études post AMM.

- Il faut pallier la restriction du champ de fabrication des équipes hospitalo-universitaires aux MTI-PP et impossibilité réglementaire pour les acteurs académiques d'obtenir un certificat BPF afin de soutenir un possible développement ultérieur à l'échelle européenne : comme pour les PTC, la fabrication et l'utilisation de MTI-PP restent couvertes par la compétence du domaine législatif et réglementaire national qui donne plus de souplesse aux établissements de santé pour valoriser leurs découvertes dans des créneaux particuliers. Dans certains cas particuliers, et pour répondre à des problématiques d'essais cliniques internationaux multicentriques, il arrive que les établissements de santé qui fabriquent des MTI-PP souhaitent bénéficier d'un certificat BPF pour permettre aux autres Etats-membres de valider les productions faites en France. La délivrance par l'ANSM d'un certificat BPF pour des établissements fabricants des MTI-PP et qui seraient conformes aux recommandations édictées par la réglementation européenne en matière de BPF ne devrait pas poser de problème.

Nota : en revanche, il est important de comprendre que la qualification BPF d'un établissement ne préjuge pas de la classification réglementaire du produit et que de ce fait, le produit restant un MTI-PP il ne devrait pas pouvoir traverser les frontières. Il faut encore une modification du cadre législatif pour parvenir à un échange intra-communautaire de ces produits, ce qui risque de prendre encore un peu de temps. En revanche, cela permettra aux chercheurs français de participer à des études dans lesquelles les patients français seront traités par des produits fabriqués en France. Les agences réglementaires sont conscientes de ce problème et de l'harmonisation nécessaire entre Etats-Membres. Du côté de la France une réflexion est en cours au sein de l'ANSM pour que des certificats BPF puissent être délivrés à des structures, qui en feraient la demande. Il est urgent, pour les installations qui répondent aux exigences PBF, que l'ANSM délivre les certificats BPF.

- Réduire les délais d'autorisation d'essais cliniques (AEC) : le délai d'autorisation moyen d'AEC pour les médicaments de thérapie cellulaire est de 138 jours en moyenne (base «Oscar» des Entreprises du Médicament [Leem]) en France, avec des extrêmes de plus de 180 jours; la réglementation européenne prévoit une durée maximale de 90 jours (renouvelable une fois). Pour les médicaments de thérapie génique, la réglementation européenne prévoit un délai de 120 jours +90 jours si nécessaire. En France, le délai d'autorisation, théoriquement de 120 jours est de 7 mois en moyenne. 
Ces délais d'évaluation plus longs en France que chez nos voisins, constituent très clairement un élément négatif pour l'attractivité de notre territoire par rapport aux pays comparables. Le nouveau règlement européen $(536 / 2014 \mathrm{CE})$ prévoit 60 jours pour ce même délai. Il est de nature à régler le problème dès lors qu'il sera mis en application sur le territoire national (au plus tôt fin mai 2016).

\section{Propositions}

Raccourcir les délais d'autorisation d'essais cliniques afin de préserver la compétitivité de notre territoire pour les thérapies innovantes. Pour cela :

- renforcer la capacité du pôle évaluation des médicaments de thérapie innovante de l'ANSM, afin que le premier délai d'évaluation ne soit pas dépassé (p. ex. 90 jours pour la thérapie cellulaire et 120 jours pour la thérapie génique);

- inciter les laboratoires demandeurs d'autorisation à présenter le futur dossier d'essai clinique aux équipes de l'ANSM lors de réunions de pré-soumission, et ce afin d'identifier en amont les faiblesses du dossier pour faciliter et accélérer l'évaluation de la demande; et

- travailler à la mise en place d'une phase pilote d'application du nouveau règlement européen (536/2014) en 2015.

\section{- Permettre le développement des médicaments fondés sur des organismes génétiquement modifiés (OGM) - une complexité supplémentaire}

La manipulation dans un but thérapeutique de cellules génétiquement modifiées ex vivo, outre de répondre aux exigences pharmaceutiques applicables aux MTI de «thérapie génique », relève également de la réglementation sur les OGM. Deux types d'utilisation confinée de ce type d'OGM sont possibles, suivant leur finalité :

- l'utilisation confinée d'OGM dans un but de recherche, de développement ou d'enseignement, régie par le décret n 2011-1177 du 23/09/2011 et donnée après avis technique et classification préalable par le Haut Conseil aux Biotechnologies (HCB) et dont l'agrément est donné par le Ministère de la Recherche et de l'Enseignement Supérieur. Délai théorique de 90 jours ;

- l'utilisation confinée d'OGM à visée industrielle, soit déclarative, soit soumise à un régime d'autorisation par le Préfet selon l'avis technique et la classification préalable donnés par le HCB - instruite et contrôlée par la Direction Régionale et Interdépartementale de l'Environnement et de l'Énergie (DRIEE) en application des prescriptions du code de l'environnement, sous l'autorité du Préfet. Le délai d'instruction de la procédure d'autorisation est de 9 à 12 mois.
Dans ce cadre réglementaire spécifiquement français, long et complexe à mettre en œuvre, ont été identifiées des barrières dont la levée accroitrait significativement la compétitivité de notre territoire pour ces activités :

1. Le mandat de 5 ans des membres du HCB arrivant à échéance au printemps 2014, le HCB devait être renouvelé. A ce jour, les nouveaux membres du HCB n'ont pas tous été nommés : aucun avis ne peut donc être valablement donné, rendant impossible l'autorisation d'essais cliniques de thérapie génique par l'ANSM depuis le mois de mai 2014 ! Par conséquent, les essais cliniques en question ne peuvent démarrer, laissant des patients atteints de maladie grave en attente de traitements expérimentaux prometteurs. Il est donc urgent que le HCB soit de nouveau opérationnel.

2. Par ailleurs, ces procédures sont longues et compliquées et elles impliquent de multiples acteurs. En particulier un axe de simplification administrative a été identifié pour les utilisations industrielles confinées des OGM dont le niveau de confinement est au maximum égal à C2L2. Dans ce cas, quand le procédé mis en œuvre a déjà été classifié par le HCB lors de demandes antérieures à visées de R\&D, alors le Préfet pourrait être dispensé de redemander l'avis et la classification par le $\mathrm{HCB}$, pour autoriser l'utilisation à visée industrielle en se fondant alors sur le document de classification du HCB émis précédemment alors porté au dossier.

\section{Propositions}

Fluidifier et certifier les procédures administratives pour l'utilisation des MTI contenant des OGM

- Nommer au plus vite le nouveau collège des membres du $\mathrm{HCB}$, et anticiper systématiquement le renouvellement des membres du HCB afin de garantir aux usagers une continuité du service.

- Dans le cadre de l'instruction des demandes d'autorisations classées pour l'environnement au titre des rubriques 2680 quand le procédé mis en œuvre a déjà été classifié par le HCB lors de demandes antérieures, autoriser le Préfet à délivrer l'utilisation à visée industrielle en se fondant sur la base du document de classification du HCB émis précédemment sans redemander un nouvel avis au HCB.

\section{- Rendre possible la mise à la disposition des cellules souches embryonnaires (ES) aux patients en routine}

Les cellules souches embryonnaires (ES) humaines, dérivées pour la première fois en 1998 par James Thomson, ${ }^{[1]}$ ont rapidement suscité un espoir considérable en matière de thérapie cellulaire. En effet, il est théoriquement possible de régénérer n'importe quel tissu in vitro à partir de ces cellules qui de surcroît 


\begin{tabular}{|l|}
\hline Article L. 2151- 3 \\
Un embryon humain ne peut être ni conçu, ni constitué par \\
clonage, ni utilisé, à des fins commerciales ou industrielles \\
Article L. 2151- 5 \\
I. \\
- Aucune recherche sur l'embryon humain ni sur les cellules \\
souches embryonnaires ne peut être entreprise sans \\
autorisation. Un protocole de recherche conduit sur un \\
embryon humain ou sur des cellules souches \\
embryonnaires issues d'un embryon humain ne peut être \\
autorisé que si : \\
1/ La pertinence scientifique de la recherche est établie \\
2/ La recherche, fondamentale ou appliquée, s'inscrit \\
dans une finalité médicale \\
3/En l'état des connaissances scientifiques, cette \\
recherche ne peut être menée sans recourir à ces \\
embryons ou ces cellules souches embryonnaires \\
4/Le projet et les conditions de mise en ouvre du \\
protocole respectent les principes éthiques relatifs à la \\
recherche sur l'embryon et les cellules souches \\
embryonnaires
\end{tabular}

Fig. 2. Extrait de la loi relative à la bioéthique. Code de la Santé Publique, principes, conditions d'autorisation des études et recherches sur l'embryon.

sont capables de s'expandre de manière illimitée. La dérivation des cellules ES humaines nécessite la destruction d'un embryon humain (5 jours post-fécondation) ce qui requiert naturellement un encadrement réglementaire de leur obtention et de leur utilisation. Cette réglementation varie largement d'un pays à un autre.

En France, la loi relative à la bioéthique de 2004, modifiée en 2011, définit ainsi les conditions légales pour pouvoir dériver, conserver et utiliser ce type cellulaire. Rédigé dans l'intention louable de proposer un «mieux-disant éthique», ces conditions imposent un grand nombre de restrictions. Par exemple, l'Article L. 2151- 5 (figure 2) impose que les projets de recherche ayant recours aux cellules souches embryonnaires « $[\ldots]$ s'inscrive[nt] dans une finalité médicale », tandis que l'article L. 21513 stipule qu' « un embryon humain ne peut être [...] utilisé à des fins commerciales ou industrielles. ». L'interprétation stricte de cette loi semble donc restreindre toute application médicale dérivée de cette recherche au champ académique non industriel, ce qui est difficilement envisageable compte tenu des coûts de développement des thérapies innovantes. En effet, parmi les essais cliniques sur les cellules souches embryonnaires humaines en cours et dans la plupart des cas les promoteurs de ceux-ci sont des sociétés privées (tableau I). Au-delà de la jurisprudence et des
Tableau I. Promoteurs des essais clinique en cours utilisant les cellules souches embryonnaires (ES).

\begin{tabular}{ll}
\hline Type de cellule & Société \\
\hline Oligodendrocytes & Geron/Asterias (USA) : \\
& NCT01217008 (2010) \\
\hline Progéniteurs & Advanced Cell Technologies (USA) : \\
Endothéliaux & NCT01469832 (2011) - \\
Rétiniens & NCT01345006 (2011)- \\
& NCT01344993 (2011) \\
& Pfizer : NCT01691261 (2012) \\
& CHA Bio\&Diostech (Corée) : \\
& NCT01674829 (2012) \\
\hline Cardiomyocytes & AP-HP (France) : \\
& NCT02057900 (2013) \\
\hline
\end{tabular}

décrets d'application qui déterminent les modalités d'application de la loi relative à la bioéthique, l'interprétation stricte de celle-ci suscite des interrogations qui pourraient être considérées comme un risque non-négligeable pour un projet industriel. Alors que la France est de facto un leader international en matière d'essais cliniques sur les cellules ES, avec le projet Transplantation of $\mathrm{Hu}$ man Embryonic Stem Cell-derived Progenitors in Severe Heart Failure (ESCORT) déjà en phase $\mathrm{I},{ }^{[11]}$ consistant à greffer des cardiomyocytes dans le cadre du traitement des ischémies, nous pouvons nous interroger sur la définition exacte qu'entend le législateur par «leur utilisation à des fins industrielles ». Dans la perspective de la création d'une filière industrielle de thérapie cellulaire, il est donc problématique que le transfert des technologies utilisant des cellules ES humaines à des partenaires industriels soit interdite. Cette contradiction représente un réel frein à la création d'une filière en France dans ce domaine, car il imposerait, pour respecter la loi, la délocalisation des unités de production et de traitement hors du territoire.

\section{Propositions}

Permettre la production pharmaceutique des médicaments basés sur les cellules ES : il conviendrait de faire évoluer le corpus législatif pour permettre, de manière encadrée, l'usage industriel de thérapies à base de cellules ES pour assurer le débouché thérapeutique des MTI concernés au bénéfice des patients alors même que les essais cliniques sont autorisés.

\subsection{Economiques et financiers}

\section{- Financement de la mise aux normes et du maintien des BPF des installations académiques}

L'application de la réglementation médicament requiert également que la fabrication des MTI-PP soit réalisée en conformité avec les «BPF médicaments ». Les hospitaliers se trouvent devant 
des problématiques spécifiques pour lesquelles ils ne sont pas formés ou qui nécessitent des moyens qu'ils ne peuvent pas toujours obtenir, compte tenu de la restriction des budgets, telle que la mise en place de normes bien plus exigeantes que le référentiel des BP tissus/cellules.

La mise en place de travaux importants et coûteux pour mettre les locaux aux normes inquiète les acteurs académiques devant l'avenir de leur travail et de leur investissement. Il est très difficile d'admettre que pendant des années une activité qui a contribué à soigner des patients puisse être interrompue.

De plus, la certification BPF ne se résume pas aux locaux, elle prend également en compte l'organisation globale du laboratoire (p. ex. personnel d'assurance qualité, qualification et validation des équipements etc.). Ainsi, le renforcement des contrôles qualité multiplie par 2 ou par 3 le coût de fabrication du médicament.

Il est clair que le niveau d'exigence qui est demandé est susceptible d'être hors de portée pour certaines structures hospitalières si des moyens ne sont pas octroyés. Il y a donc un risque que la source de produits innovants se tarisse.

\section{Propositions}

Il est nécessaire que les établissements hospitaliers possédant une structure autorisée à produire des PTC et/ou des MTI-PP prévoient une ligne budgétaire pour la mise aux normes de leurs installations et leur maintien.

\section{- Financement des essais cliniques}

Une fois les résultats précliniques in vitro et in vivo obtenus, les chercheurs hospitalo-universitaires doivent trouver euxmêmes les crédits pour réaliser les premiers essais cliniques, essentiellement par la dotation venant de programmes hospitaliers de recherche clinique (PHRC).

Le financement des essais cliniques portant sur des thérapies innovantes est onéreux, d'autant qu'elles nécessitent des suivis à long terme du fait que le produit persiste dans l'organisme (cellule ou gène) et les sources de financement académiques actuels (appels d'offre PHRC nationaux et régionaux, ANR/Direction Générale de l'Offre de Soins [DGOS] ...) ne sont pas suffisants pour mettre en œuvre les projets de recherches institutionnelles, évaluer la faisabilité des essais cliniques d'administration à l'homme et pour permettre une réelle translation des projets.

\section{Propositions}

Prévoir dans les guichets d'aide au financement public (ANR, PHRC) des appels d'offres spécifiques pour les essais portant sur des PTC et/ou MTI, avec une dotation suffisante en conséquence pour le financement des essais cliniques précoces permettant d'assurer le continuum entre la recherche académique et la recherche clinique, et la contribution à la mise aux normes BPF. Par ailleurs, s'ajoutent à ces guichets publics d'autres partenaires comme BpiFrance-financement capable de soutenir des essais cliniques précoces au travers de ses programmes de financement propres (essentiellement le programme monopartenarial d'aide pour le développement de l'innovation [ADI]) ou via les programmes qu'elle opère pour le compte de l'Etat (les programmes collaboratifs fonds unique interministériel, projets structurants pour la compétitivité et concours mondial d'innovation [ces deux derniers étant des programmes du PIA]).

\section{- Anticiper et fluidifier l'accès au marché de ces thérapies innovantes}

Aujourd'hui, les MTI sont soumis aux mêmes types d'évaluation que les autres types de médicaments. Or, ils présentent des niveaux de complexité supplémentaire liée à leur mode d'action et/ou leur pérennité dans l'organisme. Ils sont souvent conçus pour avoir un effet thérapeutique durable après des administrations uniques ou peu répétées, mais les agences fixant le prix et le remboursement ne sont pas ou peu préparées à intégrer la mesure de la persistance du « service médical rendu » dans leurs analyses.

L'extrapolation directe des modèles économiques du secteur du médicament est particulièrement difficile dans le cas des MTI autologues ou des médicaments de thérapie génique.

Dans de nombreux cas de médecine régénératrice, les MTI ont vocation à remplacer des traitements symptomatiques de soins itératifs chroniques et ces traitements MTI nécessitant une ou plusieurs administrations ont pour vocation d'être curatifs et non plus symptomatiques. Or, la persistance de l'effet à long terme ne peut être démontrée au cours de l'essai clinique (sauf à envisager des essais sur des périodes très longues) alors même que la sécurité et l'efficacité clinique du traitement le sont. Seul un suivi postAMM permettra d'apporter des données cliniques et des éléments médicaux-économiques suffisants à l'évaluation à long terme en vie réelle avec un recul significatif.

\section{Propositions}

Une concertation entre Etats-Membres concernant les méthodes d'évaluation médico-économiques applicables à ces produits représenterait un soutien considérable à cette filière. La France, compte tenu de ses capacités avérées (leader européen des essais cliniques MTI), de ses ambitions et ses infrastructures apparait être naturellement fondée à pousser et animer cette démarche, par le biais par exemple d'un G8 «thérapies innovantes » réunissant les ministres de la santé, de la recherche et de l'industrie des principaux pays européens. Même en l'absence d'harmonisation des prix et remboursements, il serait très utile que soient discutées une fréquence de ré-évaluation du «service médical rendu » afin de mesurer la persistance de l'efficacité et l'évolution du profil de sécurité des MTI dans le temps. Cette ré-évaluation 
pourrait servir de base à un échelonnage du paiement de ces médicaments, dans le cas où leur prix représenterait une contrainte budgétaire importante. Par ailleurs, rendre les MTI accessibles aux patients suppose donc une autorisation rapide de l'accès au marché, en rémunérant l'innovation et le service médical rendu, mais en le questionnant régulièrement, dans une logique de risque partagé. Le risque pour l'industriel est ainsi un manque de visibilité sur les retours financiers, péjoratif pour les investissements à venir et le développement de l'innovation en France. Le risque pour l'Etat est d'accepter au marché un produit dont le rapport bénéfice/risque à long terme et les éléments médico-économiques sont encore imparfaitement connus.

\section{Propositions}

Faire bénéficier les MTI de la procédure d'exception pour l'octroi du prix et du remboursement. Dans une logique de risque partagé entre les industriels et l'Etat, il est demandé que les médicaments de thérapies innovantes puissent bénéficier de la procédure d'exception aux modalités de fixation des prix de droit commun mise en place par le Comité Economique des Produits de Santé (CEPS) pour l'accès à l'innovation. Cette dernière permet de négocier un prix conditionnel en fonction d'indicateurs conventionnels définis dans l'accord cadre, avec la mise en place d'un suivi post-AMM dont les résultats attendus permettront d'apporter les données cliniques suffisantes à l'évaluation à plus long terme en vie réelle.

\section{- Instituer un mécanisme pérenne de prise en charge économique des PTC et MTI-PP}

Tous les projets n'ont pas vocation à aller vers l'industrialisation. Pour autant, il faut définir le modèle économique viable permettant aux patients de bénéficier des thérapeutiques innovantes que sont les PTC et les MTI-PP.

Alors que le dispositif d'autorisation nationale valable 5 ans pour les MTI-PP a été mis en place, les modalités de prise en charge économique par le système de soin sont toujours en attente de clarification

\section{Propositions}

$\mathrm{Au}$ niveau français il est nécessaire de clarifier et instituer un processus pour obtenir la prise en charge des MTI-PP et PTC par les hôpitaux soit au niveau national (HAS) soit au plan local ou régional à travers des dispositifs appropriés (ARS, budget hospitalier, missions d'enseignement, de recherche, de référence et d'innovation [MERRI recherche], missions d'intérêt général et à l'aide à la contractualisation [MIGAC], missions d'intérêt général [MIG] thérapie cellulaire ou thérapie génique... ).

\section{Conclusion}

La France a aujourd'hui entre les mains tous les atouts pour permettre un réel essor de sa filière industrielle des thérapies innovantes en émergence, porteuse de croissance économique et d'emplois. Tous les acteurs sont présents et s'efforcent de jouer activement leur rôle dans un souci d'efficacité et d'excellence. Cependant, des freins à ce développement ont été identifiés et des solutions proposées. Certaines impliquent un rôle volontariste des pouvoirs publics. L'enjeu pour l'état est de taille : éviter que se reproduise dans le domaine de la médecine régénératrice ce qui est arrivé dans le cas des médicaments contre le SIDA ou des anticorps monoclonaux : la France a été une terre de découverte mais dans laquelle ne se trouve aucune activité industrielle de production de ces médicaments?

Conflits d'intérêts. Les auteurs n'ont pas de conflits d'intérêts à déclarer en lien avec cet article.

Abréviations. ADI : aide pour le développement de l'innovation; AEC : autorisation d'essais cliniques; AFM : Association Française contre les Myopathies; AMM : autorisation de mise sur le marché; ANR : Agence Nationale de la Recherche; ANSM : Agence Nationale de Sécurité du Médicament et des Produits de Santé ; AP-HP : Assistance Publique-Hôpitaux de Paris ; ARIIS : Alliance Nationale pour les Sciences de la Vie et de la Santé; BPF : bonnes pratiques de fabrication; BPL : bonnes pratiques de laboratoire; $\mathrm{C} 4 \mathrm{C}$ : CellforCure; CAR : chimeric antigen receptor; CEA : Commissariat à l'Energie Atomique et aux Energies Alternatives; CEESP : Commission Evaluation Economique et de Santé Publique; CEPS : Comité Economique des Produits de Santé ; CHU : Centre Hospitalier Universitaire ; CIC : Centre d'Investigation Clinique; CNRS : Centre National de la Recherche; DAEC : demande d'autorisation d'essai clinique; DGOS : Direction Générale de l'Offre de Soins; DMLA : dégénérescence maculaire liée à l'âge ; DRIEE : Direction Régionale et Interdépartementale de l'Environnement et de l'Energie; EC : essai clinique; EFS : Etablissement Français du Sang; EMA : European Medicine Agency; EP : établissement pharmaceutique; FEDER : Fonds Européen de Développement Régional ; GMP : Good Manufacturing Pratice; HCB : Haut Conseil aux Biotechnologies; ICH : International Conference on Harmonisation; Inserm : Institut National de la Santé et de la Recherche Médicale ; iPS : cellules pluripotentes induites; LEEM : Les Entreprises du Médicament; MERRI : missions d'enseignement, de recherche, de référence et d'innovation; MIG : mission d'intérêt général ; MIGAC : Missions d'ntérêt général et à l'aide à la contractualisation; MTI : médicaments de thérapie innovante; MTI-PP : médicaments de thérapie innovante-préparés ponctuellement; NIH : National Institute of Health; OGM : organismes génétiquement modifiés; 
PIA : Programme d'Investissement d'Avenir; PME : petites et moyennes entreprises; PTI-PP : médicaments de thérapie innovante préparés ponctuellement; PHRC : programme hospitalier de recherche clinique ; PI : propriété intellectuelle/propriété industrielle; PCT : produits cellulaires à finalité thérapeutique; RPIB : recherches partenariales et innovation biomédicale; SATT : Sociétés d'Accélération du Transfert de Technologies; TI : transfert industriel.

\section{Références}

1. Thomson JA, Itskovitz-Eldor J, Shapiro SS, et al. Embryonic stem cell lines derived from human blastocysts. Science 1998; 282(5391): 1145-7

2. Cavazzana-Calvo M, Hacein-Bey S, de Saint Basile G, et al. Gene therapy of human severe combined immunodeficiency (SCID)-X1 disease. Science 2000; 288 (5466): 669-72

3. Yamanaka S. Strategies and new developments in the generation of patientspecific pluripotent stem cells. Cell Stem Cell 2007; 1(1): 39-49

4. Grupp SA, Kalos M, Barrett D, et al. Chimeric antigen receptor-modified $\mathrm{T}$ cells for acute lymphoid leukemia. New Engl J Med 2013; 368(16): 1509-18

5. Estimation Citigroup 2014 http: //www . citigroup. com Consulté le 25 novembre 2014

6. ANSM. Répertoires des essais cliniques de médicaments. http://ansm. sante.fr/Activites/Essais-cliniques/Repertoires-desessais-cliniques-de-medicaments/(offset)/1 Consulté le 25 novembre 2014
7. https://clinicaltrials.gov/ Consulté le 25 novembre 2014

8. Inserm. Feu vert des autorités sanitaires pour des cellules souches cardiaques. Juin 2014

http://www . inserm. fr/actualites/rubriques/actualitesrecherche/feu-vert-des-autorites-sanitaires-pour-descellules-souches-cardiaques

Consulté le 25 novembre 2014

9. Agence de la Biomédecine. 3è journées de l'Agence de Biomédecine. Mai 2013

http://www . agence-biomedecine.fr/IMG/pdf/dossier_de_ presse_abm_vdef.pdf Consulté le 25 novembre 2014 (40 pages)

10. Plan biotechnologies médicales: l'humain réparé. Mars 2014 http: //www . medicen.org/sites/default/files/uploads/plan_ indus_lhumain_repare_ami-vf.pdf Consulté le 25 novembre 2014 (4 pages)

11. Essai clinique ESCORT https://clinicaltrials.gov/ct2/show/NCT02057900 Consulté le 25 novembre 2014

Correspondance et offprints : Jérémy Magalon, Laboratoire de Culture et Thérapie Cellulaire, Hôpital de La Conception, AP-HM, 147 boulevard Baille, 13005 Marseille, France.

E-mail : jeremy.magalon@ap-hm.fr 